\title{
Does Maternal Smoking Make a Difference in Newborn Hearing Screening with Otoacoustic Emissions?
}

\author{
Jose Miguel Sequi Canet ${ }^{1 *}$, Jose Miguel Sequi Sabater ${ }^{2}$, Jose Ignacio Collar Castillo ${ }^{1}$ and Nelson Orta Sibu ${ }^{3}$ \\ ${ }^{1}$ Department of Pediatrics, Hospital Universitario Francesc de Borja, Spain \\ ${ }^{2}$ Department of Rheumatology, Hospital Universitario Reina Sofia, Spain \\ ${ }^{3}$ Visiting Professor, Hospital Universitario Francesc de Borja, Spain
}

*Corresponding author: Jose Miguel Sequi Canet, Department of Pediatrics,

Received Date: October 17, 2019

Hospital Universitario Francesc de Borja, 46701 Gandia, Spain.

Published Date: October 21, 2019

\begin{abstract}
Neonatal hearing screening by means of transient evoked otoacoustic emission (TEOAE) is widely accepted. Some studies have related how maternal smoking can be harmful for the newborn's cochlear function. The aim of this study was to investigate the relationship between maternal smoking and TEOAE newborn hearing screening results. Data were retrospectively collected from healthy vaginally delivered newborns of gestational age $>37$ weeks and body weight $>2.5 \mathrm{Kg}$, at the Francesc de Borja Hospital maternity ward in Gandia (Spain). Maternal smoking history was compared with the pass rate to TEOAE performed within the first $48 \mathrm{hrs}$. of life. Results: the study group included 12,871 newborns. In this group, no significant relation between maternal smoking and TEOAE pass rates was observed $(\mathrm{p}<0.853)$. As there were significant differences in pass rate based on gender (female better than males, $p<0.0001$ ) and feeding of newborn (breastfeeding better than formula, $p<0.0001$ ) we analyzed these subgroups separately and also found no differences related to smoking history. Conclusion: The effect of maternal smoking in newborn hearing screening with TEOAE is not significant. Further studies are needed.
\end{abstract}

Key words: Smoking; Tobacco; Otoacoustic emissions; Newborn hearing screening

\section{Introduction}

Universal newborn hearing screening is both, crucial and routinely performed because only $50 \%$ of babies born with hearing loss carry a hearing loss risk factor. Early detection leads to an efficient treatment of the affected neonates, resulting in a better final prognosis [1-3].

Several techniques are used in newborn hearing screening. Otoacoustic emissions (OAEs) are low-level acoustic signals generated by the cochlea and passed through the middle ear into the external ear canal. OAEs are an objective indication of normal cochlear function. OAEs occur in nearly all ears with normal hearing and middle ear function. Transient evoked otoacoustic emission (TEOAE) testing is one of the most frequently used techniques because of its accuracy, simplicity, speed and low cost as described in diverse studies [2,4-6].

TEOAEs are evoked across a broad frequency range when stimulated by temporally short clicks, which have broad spectral bandwidths, and multiple cochlear locations may contribute to TEOAEs measured at any specific frequency. Researchers have compared the sensitivity of evoked OAE testing with pure-tone audiometry and concluded that OAE testing is more sensitive in detecting the early onset of cochlear pathologies before a change in hearing thresholds occur [7].

A major drawback of TEOAE testing as a screening technique for newborns relates to the middle ear status, which can severely affect its pass rate. We must keep in mind that, the presence of debris and vermix in the external ear meatus of the newborn can result in false positive screenings. This factors lead to a greater than the actual hearing loss failure rate, thus a two-step newborn hearing screening protocol has been introduced in order to allow a better clearance of the middle and external ear, this means that when newborns fail TEOAE at discharge, an appointment is given to repeat the test in less than one month. This improves specificity of test without delaying adequate diagnosis.

Another crucial factor is the newborn's age when the test is done. Data strongly suggests that the prime testing window is beyond 2448 hrs. of life, as fluid in the middle ear and in the external meatus is normally significantly reduced on the second day of life. For this 
reason, the TEOAE test is done as near as possible to discharge. Spain's hospital stay average for mothers after a vaginal delivery is $48 \mathrm{hrs}$. and for cesarean section is more than $72 \mathrm{hrs}$. allowing a successful hearing screening implementation program $[2,7]$.

There are well-known hearing loss risk factors defined by the Joint Committee on Infant Hearing and The Commission for Early Detection of Hearing Loss (CODEPEH) based in Spain and others [8-10]. Newborns with these factors have a higher fail rate in TEOAEs as expected because of their hearing impairment; this is the purpose of the test.

However, some studies have demonstrated the existence of other epidemiological factors that modify TEOAE test results [11] such as gender, for example, Cassidy et al [12] concluded that female newborns present with significantly higher TEOAE levels at $1.6 \mathrm{kHz}$, $2.4 \mathrm{kHz}, 3.2 \mathrm{kHz}$, and $4.0 \mathrm{kHz}$. Also, Aidan et al. [13] demonstrated that mean TEOAE levels in female neonates are higher than in male neonates (22.1 dB SPL vs. 21.4 dB SPL). The results of experimental studies point to a potential protective effect of female sex hormones as an explanation of sex-specific differences in hearing. For example, Kilicdag et al. [14] demonstrated that estrogen therapy may protect against hearing loss in aging postmenopausal women. Research continues to determine if other related hormonal and/or neural effects have any effect.

Also breastfeeding (breastfed newborns have better response to TEOAE) can modify pass rate to hearing screening test as described in various studies [11,15-16] without a clear explanation. It is theorized that an earlier opening of the Eustachian tube and/or a better middle ear clearance based on the position of baby or the suction movements for breastfeeding can explain this.

Both factors are important elements in the TEOAE pass rate, but they act as minor risk factors. The real effect on response and pass rate of the TEOAE screening test must be considered as more data on these phenomena are gathered [17-18].

One of these additional epidemiological factors can be the tobacco effect because our knowledge regarding the harmful effects of smoking and tobacco use on hearing is still limited but increasing evidence has been accumulated on the adverse effects among different population groups [19-21].

One of these studies that link tobacco to hearing loss is Gaur's [20], in 11,454 subjects of different age-groups aged 20-60 years, where pure-tone audiometry was performed and after adjusting for other risk factors such as age and sex, current smokers were 1.69 times as likely to have hearing loss as compared to nonsmokers (CI 1.31-2.17). These findings are based in physio pathological effects of smoking that have shown to result in vascular lesions and changes in some characteristics of the blood, leading to hypoxiainduced injury of various tissues, including the hearing organ. It seems that effect is related to cochlear damage [22] because nicotine administration induced damage of the outer hair cells (OHC) which were distorted in shape with vacuolated cytoplasm and heterochromatic nuclei. Topography revealed damage of the stereocilia which included disorganization, bent and limp or complete loss and expansion of the surrounding supporting cells. These changes were more pronounced in the basal turn of the cochlea. So, nicotine has proved to be harmful to the cells of the cochlea, particularly the outer hair cells of the basal turn and this is very important because normal TEOAE results depend on outer hair cell activity.

Some other studies [23-26] confirm this effect and have suggested that tobacco and/or smoking may result in a lower otoacoustic emissions response.

Korres [25] also concluded that in utero, exposure to tobacco smoking appears to have an impact on outer hair cells. These effects seem to be equally true for all exposed newborns, regardless of the degree of exposure. According to the results of this study, smoking during pregnancy seems to influence the fetal cochlea, although the detected effects on outer hair cells were small. The adverse effects of smoking on $\mathrm{OHC}$ were measured as significantly lower TEOAE mean responses across frequencies in exposed neonates compared to non-exposed neonates. Analysis by frequency revealed significantly lower TEOAE amplitudes at $4000 \mathrm{~Hz}$. This adverse effect of smoking seemed to be equally true for newborns with low and high exposure and not as dose related as it would be expected, since by comparing subgroups with low, moderate and high exposure to each other Korres found no significant differences, neither at mean TEOAEs responses across frequencies, nor at the mean amplitudes of the frequencies tested.

Also, Vinay [27] found that TEOAE amplitudes were significantly reduced in smokers compared to non-smokers. The results found a significant effect of age on the amplitude of efferent suppression in smokers; however, no significant effect was found in the nonsmokers group.

This raises the question of whether smoking mothers can influence the newborn TEOAE screening results, in a similar manner as other previously described epidemiological factors have been shown to modify the TEOAE pass rate.

\section{Materials and Methods}

Significant differences in TEOAE amplitudes between groups can alter the pass rate of screening tests; therefore, the aim of this study was to compare maternal history of smoking with the influences on the pass rate to TEOAE test as a method for newborn hearing screening during the first $48 \mathrm{~h}$ of life.

Data were collected between 2000-2019 from all healthy newborns without any known hearing loss risk factor in the maternity ward of Francesc de Borja Hospital in Gandia (Spain). This retrospective study was approved by the ethical committee of this Hospital on 15/July/2019 with code 12/2019.

\section{Exclusion criteria}

The focus of the study was limited to healthy newborns without any syndrome or known disease. Additionally, newborns with Apgar lower than 7 at 5 min. were excluded. Only vaginally 
delivered newborns were included because timing is a crucial factor in response and neonates delivered by cesarean section stay in the hospital $72 \mathrm{hrs}$. so the TEOAE test is done around this age.

In order to eliminate other possible confounding factors [28] only newborns older than 37 gestational weeks with a birth weight greater than $2.5 \mathrm{Kg}$ were included.

\section{Protocol}

The bilateral TEOAE screening was performed as close as possible to $48 \mathrm{hrs}$. of life. Sometimes an initial TEOAE test near discharge at 48 hrs. was done even though the baby was a little fussy because the baby and the screener were both available at that moment. If the baby passed, no more testing was done. However, if the baby "failed" it is assumed it was because the baby was fussy and another test was done a few hours later, immediately before discharge, most of these babies passed the test. Thus, in this case, the second test was the more valid result and was the one included in this study.

All nurses performed the screening, on every shift, every day of the week, depending on availability. The screening was performed in the newborn room with as little background noise as possible after parental verbal consent was obtained. Testing usually took place after feeding time to ensure the newborn was calm. No sedation was administered.

\section{Techniques}

The TEOAEs were recorded with an ECHOCHECK OAE Screener ${ }^{\circledR}$ based on the IL088 (Otodynamics Ltd. Hatfield, U.K.) system and connected to the ILO ECP $®$ neonatal probe. This emits a simultaneous standard click-type non-linear stimulus of $1 \mathrm{~ms}$ duration. The intensity of which is $84 \pm 3 \mathrm{~dB}$ SPL (sound pressure level) 80 times per second and receives and averages the responses issued by the cochlea to OAEs from $1-4 \mathrm{kHz}$ with a primary response band of 1.6-3.2 kHz., but with the frequency $1.6 \mathrm{kHz}$ filtered to avoid noise contamination.

The device is small and portable. Its measurement automatically adapts to the size of the external auditory canal. It has luminous signals that confirm that the stimulus is reaching the ear correctly and that the noise level is admissible for the test (less than 47.3 $\mathrm{dB}$ SPL on average, although in certain frequencies may be higher). "Pass" results indicates there are TEOAEs. A normal result (pass) requires a signal/noise level response above $6 \mathrm{~dB}$ with a minimum of 512 valid responses for at least $5 \mathrm{sec}$. The duration of the test usually oscillates between $45 \mathrm{sec}$ and a maximum of $5 \mathrm{~min}$.

A newborn with normal bilateral response was accepted as a pass; otherwise it was deemed a referral [29].

\section{Statistical analysis}

The dependent variable is the TEOAE result before discharge at $48 \mathrm{hr}$. of life (pass/refer).

The independent variable is smoking during pregnancy registered in maternal history (yes/no).
Additionally, differences in pass rate to TEOAE test by gender or feeding were analyzed and also between derived subgroups versus smoking history.

Following frequency analysis of the variables, a univariate analysis was completed between the TEOAE results and the study variables with the Chi-squared test and risk estimate with odds ratio.

Statistical analyses were only conducted on patients that had data available for either of the study variables (Maternal smoking vs TEOAE pass/refer).

The significance level was established at $\mathrm{p}<0.05$. The data were analyzed using Excel® 2016 and SPSS $®$ version 20.

\section{Results}

(Tables 1-11).

Table 1: Mothers that declared to be smokers during pregnancy.

\begin{tabular}{|c|c|c|c|}
\hline \multirow{2}{*}{ Smoking mothers } & & Frequency & Valid Percent \\
\hline & No & 10267 & 80.1 \\
\hline Total & Yes & 2543 & 19.9 \\
\hline Missing & & 12810 & 100 \\
\hline Total & & 61 & \\
\hline
\end{tabular}

Table 2: Results for newborn hearing screening with TEOAE.

\begin{tabular}{|c|c|c|c|}
\hline \multirow{2}{*}{ Result } & & Frequency & Valid Percent \\
\hline & TEOAE FAIL & 979 & 7.7 \\
\cline { 2 - 4 } & TEOAE PASS & 11743 & 92.3 \\
\hline Total & & 12722 & 100 \\
\hline Missing & & 149 & \\
\hline Total & & 12871 & \\
\hline
\end{tabular}

Table 3: Crosstab smoking mothers vs TEOAE results.

\begin{tabular}{|c|c|c|c|c|}
\hline & & \multicolumn{3}{|c|}{ TEOAE } \\
\hline & & TEOAE Fail & TEOAE Pass & Total \\
\hline \multirow[t]{2}{*}{ Smoking mother } & No & 777 & 9384 & 10161 \\
\hline & Yes & 194 & 2307 & 2501 \\
\hline Total & & 971 & 11691 & 12662 \\
\hline Pearson Chi-Square & $\mathrm{p}$ & 0.853 & & \\
\hline \multicolumn{2}{|l|}{ Risk Estimate } & \multicolumn{3}{|c|}{ 95\% Confidence Interval } \\
\hline & Value & Lower & Upper & \\
\hline $\begin{array}{l}\text { Odds Ratio for smoking } \\
\text { mother (No / Yes) }\end{array}$ & 0.985 & 0.836 & 1.16 & \\
\hline
\end{tabular}

Table 4: Gender of newborns.

\begin{tabular}{|c|c|c|c|}
\hline \multirow{2}{*}{ Gender } & & Frequency & Valid Percent \\
\cline { 2 - 4 } & Female & 6387 & 49.7 \\
\hline \multirow{2}{*}{ Total } & & 6466 & 50.3 \\
\hline Missing & & 12853 & 100 \\
\hline Total & & 18 & \\
\hline
\end{tabular}


Table 5: Crosstab gender vs TEOAE results.

\begin{tabular}{|c|c|c|c|c|}
\hline & & \multicolumn{3}{|c|}{ TEOAE } \\
\hline & & TEOAE Fail & TEOAE Pass & Total \\
\hline \multirow[t]{2}{*}{ Gender of newborns } & Female & 427 & 5890 & 6317 \\
\hline & Male & 551 & 5837 & 6388 \\
\hline Total & & 978 & 11727 & 12705 \\
\hline Pearson Chi-Square & $\mathrm{p}$ & 0.0001 & & \\
\hline \multicolumn{2}{|l|}{ Risk Estimate } & \multicolumn{3}{|c|}{ 95\% Confidence Interval } \\
\hline & Value & Lower & Upper & \\
\hline $\begin{array}{l}\text { Odds Ratio for gender } \\
\text { (Female / Male) }\end{array}$ & 0.768 & 0.673 & 0.876 & \\
\hline
\end{tabular}

Table 6: Females: Crosstab smokers vs TEOAE.

\begin{tabular}{|c|c|c|c|c|}
\hline & & \multicolumn{3}{|c|}{ TEOAE } \\
\hline & & TEOAE Fail & TEOAE Pass & Total \\
\hline \multirow[t]{2}{*}{ Smoking mother } & No & 340 & 4658 & 4998 \\
\hline & Yes & 84 & 1205 & 1289 \\
\hline Total & & 424 & 5863 & 6287 \\
\hline Pearson Chi-Square & $\mathrm{p}$ & 0.715 & & \\
\hline \multicolumn{2}{|l|}{ Risk Estimate } & \multicolumn{3}{|c|}{ 95\% Confidence Interval } \\
\hline & Value & Lower & Upper & \\
\hline $\begin{array}{l}\text { Odds Ratio for smoking } \\
\text { mother (No / Yes) }\end{array}$ & 1.047 & 0.818 & 1.341 & \\
\hline
\end{tabular}

Table 7: Males: Crosstab smokers vs TEOAE.

\begin{tabular}{|c|c|c|c|c|}
\hline & \multicolumn{3}{|c|}{ TEOAE } \\
\hline & & TEOAE Fail & TEOAE Pass & Total \\
\hline \multirow[t]{2}{*}{ Smoking mother } & No & 436 & 4714 & 5150 \\
\hline & Yes & 110 & 1098 & 1208 \\
\hline Total & & 546 & 5812 & 6358 \\
\hline Pearson Chi-Square & $\mathrm{p}$ & 0.475 & & \\
\hline \multicolumn{2}{|l|}{ Risk Estimate } & \multicolumn{3}{|c|}{ 95\% Confidence Interval } \\
\hline & Value & Lower & Upper & \\
\hline $\begin{array}{l}\text { Odds Ratio for smoking } \\
\text { mother (No / Yes) }\end{array}$ & 0.923 & 0.742 & 1.149 & \\
\hline
\end{tabular}

Table 8: Newborn feeding type.

\begin{tabular}{|c|c|c|c|}
\hline & & Frequency & Valid Percent \\
\hline \multirow{2}{*}{ Feeding } & Formula & 3469 & 27 \\
\cline { 2 - 4 } & Breast & 9397 & 73 \\
\hline \multirow{2}{*}{ Total } & & 12866 & 100 \\
\hline Missing & & 5 & \\
\hline Total & & 12871 & \\
\hline
\end{tabular}

Table 9: Crosstab feeding vs TEOAE results.

\begin{tabular}{|c|c|c|c|c|}
\hline & \multicolumn{3}{|c|}{ TEOAE } \\
\hline & & TEOAE Fail & TEOAE Pass & Total \\
\hline \multirow[t]{2}{*}{ Newborn feeding } & Formula & 331 & 3083 & 3414 \\
\hline & Breast & 648 & 8658 & 9306 \\
\hline Total & & 979 & 11741 & 12720 \\
\hline Pearson Chi-Square & $\mathrm{p}$ & 0.0001 & & \\
\hline \multicolumn{2}{|c|}{ Risk Estimate } & \multicolumn{3}{|c|}{ 95\% Confidence Interval } \\
\hline & Value & Lower & Upper & \\
\hline $\begin{array}{l}\text { Odds Ratio for } \\
\text { smoking mother (No } \\
\text { / Yes) }\end{array}$ & 1.434 & 1.249 & 1.648 & \\
\hline
\end{tabular}

Table 10: Only Formula: Crosstab Smokers vs TEOAE.

\begin{tabular}{|c|c|c|c|c|}
\hline & & \multicolumn{3}{|c|}{ TEOAE } \\
\hline & & TEOAE Fail & TEOAE Pass & Total \\
\hline \multirow[t]{2}{*}{ Smoking mother } & No & 220 & 2150 & 2370 \\
\hline & Yes & 109 & 913 & 1022 \\
\hline Total & & 329 & 3063 & 3392 \\
\hline Pearson Chi-Square & $\mathrm{p}$ & 0.212 & & \\
\hline \multicolumn{2}{|l|}{ Risk Estimate } & \multicolumn{3}{|c|}{ 95\% Confidence Interval } \\
\hline & Value & Lower & Upper & \\
\hline $\begin{array}{l}\text { Odds Ratio for smoking } \\
\text { mother (No / Yes) }\end{array}$ & 0.857 & 0.673 & 1.092 & \\
\hline
\end{tabular}

Table 11: Breastfeeding: Crosstab Smokers vs TEOAE.

\begin{tabular}{|c|c|c|c|c|}
\hline & & \multicolumn{3}{|c|}{ TEOAE } \\
\hline & & TEOAE Fail & TEOAE Pass & Total \\
\hline \multirow[t]{2}{*}{ Smoking mother } & No & 557 & 7233 & 7790 \\
\hline & Yes & 85 & 1393 & 1478 \\
\hline Total & & 642 & 8626 & 9268 \\
\hline Pearson Chi-Square & $\mathrm{p}$ & 0.052 & & \\
\hline \multicolumn{2}{|l|}{ Risk Estimate } & \multicolumn{3}{|c|}{$\mathbf{9 5 \%}$ Confidence Interval } \\
\hline & Value & Lower & Upper & \\
\hline $\begin{array}{l}\text { Odds Ratio for smoking } \\
\text { mother (No / Yes) }\end{array}$ & 1.262 & 0.997 & 1.597 & \\
\hline
\end{tabular}

\section{Discussion}

In this study $19.9 \%$ of mothers stated that they were smokers (Table 1). The prevalence of smoking varies greatly between countries and this figure seems to be a high percentage compared with some reviews concluding that approximately $10 \%$ of women report smoking during pregnancy but similar to others which state that the prevalence of smoking among pregnant women ranges from $23 \%$ to $25 \%$. As our figures are self-reported smoking, we think they were real but could be higher. A study on the veracity of the perinatal survey responses showed that up to $4 \%$ of mothers had cotinine in their urine, despite stating they were non-smokers, which suggests they did not respond truthfully or were passive smokers [30-31].

The mean failure rate to TEOAE test before discharge was 7.7\%. This is a normal figure $[1,2,5,7]$ for first step tests in good newborn hearing protocols (Table 2).

The main result of this study is that no significant differences were found in pass rate to first TEOAE test between smoking mothers and non-smoking ones (Table 3).

This is a very interesting result, since some studies have related smoking with hearing loss and TEOAE test fails if there is a hearing loss greater than $30 \mathrm{~dB}$ HL. From our study, one can conclude that smoking during pregnancy did not greatly affect the newborn's cochlear response or not so much to have more than $30 \mathrm{~dB}$ HL hearing loss and alter pass rate to newborn hearing screening programs.

These results disagree with our expected hypothesis, based in different studies, which insist in the relation between smoking and hearing loss in infancy. A recent review by Vasconcellos [24] 
concluded that even low levels of passive tobacco exposure, both in utero and from secondhand smoke in childhood, pose a risk for pediatric sensorineural hearing loss (SNHL). Thus, reduction without cessation seems insufficient to eliminate tobaccoassociated risk both during pregnancy and afterward. This is since in utero exposure, even if the mother quits smoking during the first trimester of pregnancy, may be injurious to the still developing auditory system, which during the first trimester is reaching its complete functionality [18].

This can be the reason for the association between maternal smoking during pregnancy and later hearing loss, even though a substantial percentage of women reported quitting smoking sometime during the pregnancy. This implies that even brief smoke exposure during the first trimester of pregnancy has farreaching consequences for hearing. Prenatal smoke exposure is independently being associated with higher pure-tone hearing thresholds and an almost 3-fold increase in the odds of unilateral low-frequency hearing loss among adolescents [23].

The rationale for using TEOAE test in our study is that the effect of smoking (nicotine) on cochlear health and function has been well documented and these changes were more pronounced in the basal turn of the cochlea involving mainly the outer hair cells (responsible for otoacoustic emissions) [22]. One explanation for this could be the extreme sensitivity of the outer hair cells to hypoxia, since it is known that nicotine produces a vasospasm which reduces the amount of oxygen brought to the cochlea. In addition, there is increased blood viscosity which would cause cochlear damage and also reduce otoacoustic emission response [32].

This can be the reason why specific studies, like Durante's [31] which compares the effect of smoking on newborns' otoacoustic emissions, shows that their intensity is lower if mothers smoked during pregnancy with a mean difference between the groups of $2.47 \mathrm{~dB}$ sound pressure level (SPL), regardless of the number of cigarettes per day. Other studies in neonates have also shown that prenatal exposure to tobacco smoke may impair cochlear function $[25,31]$ and disrupt auditory brainstem responses [33].

The fetus could be particularly sensitive to this toxin because, according to Korres et al. [25] nicotine easily enters the placental barrier and may reach fetal plasma concentration in levels $15 \%$ higher than those in the mother. In addition, the concentration in amniotic fluid is also higher, up to $54 \%$ higher than that of the mother, and the concentration in the fetus therefore increases from swallowing the fluid. This increases the effect on the outer hair cells, which also have direct nicotinic acetylcholine receptors, adding to the hypoxic effect of the previously mentioned vasoconstriction.

Our study's main hypothesis was based on all these data, but results did not show differences between the newborns studied. This can perhaps be explained because a lower amplitude of TEOAE with tobacco consumption can be insufficient to get a "fail/refer" on newborn hearing screening tests because neonates have such a powerful response to the TEOAE test that can offset the differences. That is a very interesting practical question for newborn hearing screening. In fact, in Korres 's study mean TEOAEs response across frequency for exposed newborns was $19.5 \mathrm{~dB}$ SPL, and for nonexposed neonates was just $20.6 \mathrm{~dB}$ SPL; signal to noise ratio analysis by frequency demonstrated similar TEOAE mean amplitudes for all but one of the examined frequencies $(4.000 \mathrm{~Hz})$. The TEOAE test needs a $30 \mathrm{~dB}$ HL hearing loss to get a "refer", so perhaps tobacco slightly diminishes the response but is not of clinical interest because it is not responsible for a significant increase in percentage of fails in newborn hearing screening protocols.

In this study $50.3 \%$ of newborns were males (Table 4). The differences in TEOAEs depending on gender are well known and this study confirms them as very significant differences in TEOAE pass rates were evidenced with $\mathrm{p}<0.0001$ (Table 5). For this reason, this study separately analyzed gender subgroups with tobacco consumption/TEOAE results and found no significant differences in any subgroup (Tables 6-7).

The rationale for analyzing gender influences is based on the studies aforementioned in the introduction [11-15] and also on studies like the one from Lisowska G [26] on 84 healthy volunteers that concluded that smoking does not modulate a hearing threshold determined with pure tone audiometry at low, moderate, and ultrahigh frequencies, but causes a significant decrease in OAE levels. This effect was observed only in males, which implies that they are more susceptible to smoking-induced hearing impairment. Sex-specific differences in otoacoustic emissions level may reflect influences of genetic, hormonal, behavioral, and/or environmental factors. Taking the evidence of Kilicdag [14] into account, it can be hypothesized that female sex hormones may also protect against tobacco smoke-induced hearing impairment, but our study did not show any differences based on newborn gender and smoking mothers/TEOAE results, thus more studies are needed.

In our study $73 \%$ of newborns were breastfed (Table 8). The rationale for analyzing feeding depends on the findings that breastfed newborns have better pass rate to TEOAE test than formula-fed ones $[11,15,16]$. Our study confirms this very significant difference $(\mathrm{p}<0.0001)$, having a real effect in the pass rate to the test and therefore it can be a possible confounding factor for tobacco effect (Table 9). The result of our study showed no differences when we analyzed separately by feeding groups the effect of mothers' smoking history and pass rate to TEOAE (Tables 10\&11).

This is an important conclusion because after birth, breastmilk is also a potential source of nicotine exposure. Some studies have shown that nicotine and cotinine can get concentrations two to three times higher in breast milk than in plasma [30,34]. Cotinine is a metabolite of nicotine, commonly used as biomarker of exposure to tobacco smoke. It has a half-life of approximately 16 to 20 hours and, therefore, reflects tobacco smoke exposure within the past 2 or 3 days. Moreover, nicotine and cotinine peaked in the breast milk at $0.5 \mathrm{hrs}$. after just one cigarette smoked. The nicotine disappeared from the milk by $3 \mathrm{hrs}$. while the cotinine required $72 \mathrm{hrs}$. The relative infant doses of nicotine and cotinine were found to be, $12.8 \%$ and $77.6 \%$, respectively [35]. 
This raises the question of whether newborns from smoking mothers that are being breastfed can have a higher fail rate than formula-fed ones. However, in our study, no differences in feeding subgroups were found.

In light of these results, the absence of statistical differences can be related to the fact that while staying in hospital, smoking is forbidden and the effect of nicotine is short, so in the two days after delivery no direct effect depending on feeding must be expected. Another possible explanation is that the positive effect of breastfeeding in newborn's TEOAEs compensates the negative effect of tobacco.

In our study, the breastfed newborns subgroup was nearly significant $(\mathrm{p}<0.052)$ but with an unexpected higher ratio (OR 1.26) of "fail/refer" in non-smoking mothers. This result for us is unexplainable and lacks common sense based on the discussions above. We think it can be a spurious statistical result until new studies confirm so. Considering this result, we must bear in mind that a possible explanation could be that passive smoking at home is also associated with measurable nicotine levels in breastmilk [36]. Unfortunately, our study did not collect that information and we are unable to confirm this hypothesis, but this raises another question on whether the possible effect of passive smoking (fathers who smoke but mothers who do not) is worth discussing.

There are some studies which conclude that exposure to tobacco smoke is associated with hearing loss in adults [32] but studies of the association between passive smoking and hearing loss in children are limited.

Wilunda et al [37] in a study that analyzed postnatal exposure to tobacco concluded that in this retrospective cohort of 50,734 children, comparing children exposed with children not exposed to tobacco smoke prenatally and at 4 months, the risk of hearing impairment was elevated in children exposed to only maternal past smoking during pregnancy (RR 1.26), only second-hand smoke at 4 months (RR 1.30), only smoking during pregnancy (RR 1.68) and smoking during pregnancy and second-hand smoke at 4 months (RR 2.35). This study had a strong limitation, however, because screening for hearing impairment was done by the mother using the whispered voice test and its validity when administered by mothers is unknown.

Three other cross-sectional studies showed that smoking during pregnancy and exposure to environmental tobacco smoke are associated with sensorineural hearing impairment in 5-11-year old's and teenagers [38-40]. Besides being cross-sectional, these studies were based on small samples and adjusted for only a few potential confounders. Moreover, the individual and joint effects of prenatal and postnatal exposure to tobacco smoke on hearing impairment in children are yet to be explored. Thus, more evidence from cohort studies is needed.

Another important factor to be considered in future studies is the correlation between exposure to cigarette smoking and the good function of the Eustachian tube, which was thoroughly analyzed by Pezzoli et al [41]. They found the presence of a high number of smokers suffering from tubal dysfunction. This has important clinical relevance in a study like ours because smoking increases the incidence of middle ear diseases and this event can impair the results in TEOAE test.

Despite all of the data discussed above, not all studies agree on the harmful effects of tobacco in hearing acuity. Nomura et al. [32] conducted a meta-analysis of 15 studies published between 1966 and 2003, in order to determine the effect of cigarette smoking on the results of audiometry and documented the unfavorable effect of smoking in 9 out of the 15 analyzed studies; the lack of such an association in the remaining studies suggests that the relationship between smoking and hearing impairment is still not completely understood.

For example, in adults, Nondahl [42] analyzed serum cotinine in a total of 197 participants with incident hearing loss and 394 control participants, aged 53 to 75 years, selected from the 2,800 participants of the 5-year follow-up examination of the populationbased "Epidemiology of Hearing Loss Study 1998-2000" and found no significant associations between serum cotinine levels and incident hearing loss.

Some other adult studies have concluded something similar like the Framingham Study where authors assessed low-frequency (250- to $1000-\mathrm{Hz}$ ) and high-frequency (4000- to $8000-\mathrm{Hz}$ ) hearing loss among 1,662 study participants and found no association. The Baltimore Longitudinal Study of Aging also found no association between baseline smoking habits and the incidence of hearing loss at 500 to $3,000 \mathrm{~Hz}$ among 531 men, although with only 46 incident cases of hearing loss there may have been limited power to detect an association [43-44]. And Johnson's study [45] in a cohort of male twins, including 583 individuals (239 twin pairs and 105 individuals without their twin brother) also concluded that no increased risk for hearing loss was seen in smokers when compared to non-smokers.

In children there are some studies with similar conclusions, like Butcher's [17] study about risk factors for permanent childhood hearing loss on a large series of 19,504 non-increased hearing risk children, who concluded that there is no association between maternal report of smoking before or during pregnancy and hearing loss, OR: 0.57 (0.23 to 1.42).

As far as we know, there is only one study similar to ours from Islek Seçen in 919 mothers that is also consistent with our results because it showed no differences between smoking and nonsmoking mothers on newborns otoacoustic emission test results [46]. This makes our study more credible, but more similar studies are needed to confirm these results before drawing a reliable conclusion.

\section{Limitations}

The Echocheck Screener results do not provide actual TEOAE response amplitude values. As stated by other authors above, it is possible that tobacco exposed newborns may have reduced 
amplitude OAE responses compared with non-exposed groups. Although the responses may be significantly lower, those could still be adequate for newborns to pass the screening test. The TEOAE test without normal results indicates a hearing loss greater than 30 dB HL. Perhaps smoking during pregnancy did not greatly alter the function of the hearing organ to cause a $30 \mathrm{~dB}$ HL hearing loss and cause a "refer" in TEOAE test. This study cannot conclude that there is not any mild difference in OAE response amplitudes across the whole group. Additional studies using actual response amplitude data are needed to consider this latter point.

The nonlinear protocol used in the current study is the most common method to record TEOAEs [29]. This method uses three clicks of one polarity with a subsequent single click with three times the amplitude and opposite polarity. The test can detect cochlear responses in the presence of linear artifacts related to the clicks. However, part of the actual OAE recording is eliminated as all linear components of the response are removed. Therefore, nonlinear measurement may not be able to detect the OAE response completely; this process results in a low signal-to-noise ratio of TEOAEs in general and may possibly contribute to the lack of amplitude difference among groups, thus not altering the pass rate. Perhaps it is necessary that linear measurement of TEOAEs should also be recorded in addition to using a nonlinear protocol in order to clarify this issue in future research.

The Echocheck Screener explores a frequency range from 0 to 4 $\mathrm{kHz}$. Further studies are required to determine if there is any effect in some of the frequencies outside of this range, such as differences in higher frequencies that cannot be detected with this device.

Given that healthy newborns were examined for this study, it remains unknown if tobacco exposure increases the susceptibility to other neonatal hearing loss factors such as hypoxia or ototoxic drugs adding risk and lowering TEOAE response in the newborns. More studies in this area are needed.

Additionally, perhaps there are other unknown perinatal factors stronger than tobacco exposure that can mask their effect if the influence is mild in nature.

Failing the TEOAE test reflects hearing loss greater than 30 $\mathrm{dB}$ HL; however, there are other factors that can alter the results, including the high frequency of the etiology not being in the inner ear but in the middle ear or in the external meatus. The findings make it cumbersome to pair the test result to actual cochlear response, however, given the large sample size, and the fact that all the newborns in the study were healthy, it can be assumed that in order to pass the test, the influence of middle ear status was similar for all.

This study did not record the amount of tobacco consumed by mothers, that can be an issue to address. This study relies on maternal history and some mothers can lie when asked questions about smoking habits. This is probably not a major issue since the aforementioned studies of Korres and Durante did not show differences on TEOAE amplitude depending on number of cigarettes smoked per day. Our study also did not take into account secondhand exposure to tobacco that can be harmful and associated with increased incidence of hearing loss directly related to level of exposure [47], in this case more studies are also needed.

\section{Conclusion}

Maternal smoking appears not to be a significant factor in the pass rate of TEOAE screening in healthy term newborns that are vaginally delivered. Analyzed separately, neither gender nor feeding type changed these results. That can be interesting for newborn hearing screening programs. Additional studies are needed to clarify this issue and if there is any mild effect in the response amplitude that is not significant enough to make a "test refer", or if it affects frequencies not explored by the Echocheck screener.

\section{Contribution}

Conceptualization: Sequi Canet JM and Sequi Sabater JM; Data curation: Collar Castillo J; Supervision: Orta Sibu N.

\section{Funding}

This research received no external funding.

\section{Acknowledgment}

Thanks to all pediatric department in Hospital Francesc Borja Gandia.

\section{Conflicts of Interest}

The authors declare no conflict of interest.

\section{References}

1. Cubells Fuente JM, Gairí Tahull JM (2000) Neonatal screening for deafness through evoked acoustic broadcasts. An Pediatrics 53(6): 586591.

2. Sequi Canet JM, Sala Langa MJ, Collar del Castillo JI (2016) Results from ten years newborn hearing screening in a secondary hospital. An Pediatr 85(4): 189-196.

3. Neumann K, Chadha S, Tavartkiladze G, Bu X, White KR (2019) Newborn and Infant Hearing Screening Facing Globally Growing Numbers of People Suffering from Disabling Hearing Loss. Int J Neonatal Screen 5(1): 7.

4. Wong LY, Espinoza F, Alvarez KM, Molter D, Saunders JE (2017) Otoacoustic Emissions in Rural Nicaragua: Cost Analysis and Implications for Newborn Hearing Screening. Otolaryngol Head Neck Surg 156(5): 877-885.

5. Akinpelu OV, Peleva E, Funnell WR, Daniel SJ (2014) Otoacoustic emissions in newborn hearing screening: a systematic review of the effects of different protocols on test outcomes. Int J Pediatr Otorhinolaryngol 78(5): 711-717.

6. Chiou ST, Lung HL, Chen LS, Yen AM, Fann JC, et al. (2017) Economic evaluation of long-term impacts of universal newborn hearing screening. Int J Audiol 56(1): 46-52.

7. Sequí Canet JM, Collar del Castillo J, Lorente Mayor L, Oller Prieto A, Morant Barber M, et al. (2005) Organization of neonatal auditory screening with otoemissions in secondary hospitals: feasible, efficient and effective. Acta pediatr Esp 63(11): 465-470.

8. American Academy of Pediatrics, Joint Committee on Infant Hearing (2007) Year 2007 position statement: principles and guidelines for early hearing detection and intervention programs. Pediatrics 120(4): 898921.

9. Onoda RM, Azevedo MF, Santos AM (2011) Neonatal Hearing Screening: 
Failures, hearing loss and risk indicators. Braz J Otorhinolaryngol 77(6): 775-783.

10. Trinidad-Ramos G, de Aguilar VA, Jaudenes-Casaubón C, Núñez-Batalla F, Sequí-Canet JM Comisión para la Detección Precoz de la Hipoacusia (CODEPEH) (2010) Early hearing detection and intervention: 2010 CODEPEH recommendation. Acta Otorrinolaringol Esp 61(1): 69-77.

11. Sequi-Canet JM, Sala-Langa MJ, Collar del Castillo JI (2014) Perinatal factors affecting the detection of Otoacoustic Emissions in vaginally delivered, healthy newborns, during the first 48 hours of life. Acta Otorrinolaringol 65(1): 1-7.

12. Cassidy JW, Ditty KM (2001) Gender differences among newborns on a transient otoacoustic emissions test for hearing. J Music Ther 38(1): 2835.

13. Aidan D, Lestang P, Avan P, Bonfils P (1997) Characteristics of transientevoked otoacoustic emissions (TEOES) in neonates. Acta Otolaryngol $117(1): 25-30$.

14. Kilicdag EB, Yavuz H, Bagis T, Tarim E, Erkan AN, et al. (2004) Effects of estrogen therapy on hearing in postmenopausal women. Am J Obstet Gynecol 190(1): 77-82.

15. Van Kerschaver E, Boudewyns AN, Declau F, van de Heyning PH, Wuyts FL (2013) Socio-demographic determinants of hearing impairment studied in 103835 term babies. Eur J Public Health 23(1): 55-60.

16. Garcia MV, Azevedo MF, Testa JR, Luiz CB (2012) The influence of the type of breastfeeding on middle ear conditions in infants. Braz J Otorhinolaryngol 78(1): 8-14.

17. Butcher E, Dezateux C, Knowles RL (2018) Risk factors for permanent childhood hearing impairment. Arch Dis Child 0: 1-3.

18. Roizen N (2003) Non genetic causes of hearing loss. Ment Retard Dev Disabil Res Rev 9(2): 120-127.

19. Aligne CA, Stoddard JJ (1997) Tobacco and children. An economic evaluation of the medical effects of parental smoking. Arch Paediatr Adolesc Med 151(7): 648-653.

20. Gaur K, Kasliwal N, Gupta R (2012) Association of smoking or tobacco use with ear diseases among men: a retrospective study. Tob Induc Dis 10(1): 4

21. Cruickshanks KJ, Klein R, Klein BE, Wiley TL, Nondahl DM, et al. (1998) Cigarette smoking and hearing loss: the epidemiology of hearing loss study. JAMA 279(21): 1715-1719.

22. Amel MM, Abdel-Hafez, Sanaa AM Elgayar, Ola A Husain, Huda SA Thabet (2014) Effect of nicotine on the structure of cochlea of guinea pigs. Anat Cell Biol 47(3): 162-170.

23. Weitzman M, Govil N, Hua Liu Y, Lalwani AK (2013) Maternal Prenatal Smoking and Hearing Loss Among Adolescents. JAMA Otolaryngol Head Neck Surg 139(7): 669-677.

24. Vasconcellos AP, Kyle ME, Gilani S, Shin JJ (2014) Personally Modifiable Risk Factors Associated with Pediatric Hearing Loss: A Systematic Review. Otolaryngol Head Neck Surg 151(1): 14-28.

25. Korres S, Riga M, Balatsouras D, Papadakis CH, Kanellos P, et al. (2007) Influence of smoking on developing cochlea. Does smoking during pregnancy affect the amplitudes of transient evoked otoacoustic emissions in newborns? Int J Pediatr Otorhinolaryngol 71(5): 781-786.

26. Lisowska G, Jochem J, Gierlotka A, Misiołek M, Ściersk W (2017) SexRelated Cochlear Impairment in Cigarette Smokers. Med Sci Monit 23: 377-397.

27. Vinay (2010) Effect of smoking on transient evoked otoacoustic emissions and contralateral suppression. Auris Nasus Larynx 37(3): 299-302.

28. Cavalcante JM, Isaac Mde L (2013) Analysis of otoacoustic emissions in neonates at term and preterm. Braz J Otorhinolaryngol 79(5): 582-588.
29. Kemp DT, Ryan S, Bray P (1990) A Guide to the Effective Use of Otoacoustic Emissions. Ear Hear 11(2): 93-105.

30. Napierala M, Mazela J, Merritt TA, Florek E (2016) Tobacco smoking and breastfeeding: Effect on the lactation process, breast milk composition and infant development. A critical review. Environ Res 151: 321-338.

31. Durante AS, Ibidi SM, Lotufo JP, Carvallo RM (2011) Maternal smoking during pregnancy: impact on otoacoustic emissions in neonates. Int J Pediatr Otorhinolaryngol 75(9): 1093-1098.

32. Nomura K, Nakao M, Morimoto T (2005) Effect of smoking on hearing loss: quality assessment and meta-analysis. Prev Med 40(2): 138-144.

33. Kable JA, Coles CD, Lynch ME, Carroll J (2009) The impact of maternal smoking on fast auditory brainstem responses. Neurotoxicol Teratol 31(4): 216-224.

34. Luck W, Nau H (1985) Nicotine and cotinine concentrations in serum and urine of infants exposed via passive smoking or milk from smoking mothers. J Pediatr 107(5): 816-820.

35. Calvaresi V, Escuder D, Minutillo A, Bastons-Compta A, García-Algar 0, et al. (2016) Transfer of Nicotine, Cotinine and Caffeine Into Breast Milk in a Smoker Mother Consuming Caffeinated Drinks. J Anal Toxicol 40(6): 473-477.

36. Dahlström A, Ebersjö C, Lundell B (2004) Nicotine exposure in breastfed infants. Acta Paediatr 93(6): 810-816.

37. Wilunda C, Yoshida S, Tanaka S, Kanazawa Y, Kimura T, et al. (2018) Exposure to tobacco smoke prenatally and during infancy and risk of hearing impairment among children in Japan: A retrospective cohort study. Paediatr Perinat Epidemiol 32(5):430-438.

38. Lalwani AK, Liu YH, Weitzman M (2011) Secondhand smoke and sensorineural hearing loss in adolescents. Arch Otolaryngol Head Neck Surg 137(7): 655-662.

39. Talaat HS, Metwaly MA, Khafagy AH, Abdelraouf HR (2014) Does passive smoking induce sensorineural hearing loss in children? Int J Pediatr Otorhinolaryngol 78(1): 46-49.

40. Weitzman M, Govil N, Liu YH, Lalwani AK (2013) Maternal prenatal smoking and hearing loss among adolescents. JAMA Otolaryngol Head Neck Surg 139(7): 669-677.

41. Pezzoli M, Lofaro D, Oliva A, Orione M, Cupi D, et al (2017) Effects of Smoking on Eustachian Tube and Hearing. Int Tinnitus J 21(2): 98-103.

42. Nondahl DM, Cruickshanks KJ, Dalton DS, Schubert CR, Klein BE, et al. (2004) Tweed TS. Serum Cotinine Level and Incident Hearing Loss A Case-Control Study. Arch Otolaryngol Head Neck Surg 130(11): 12601264.

43. Gates GA, Cobb JL, D'Agostino RB, Wolf PA (1993) The relation of hearing in the elderly to the presence of cardiovascular disease and cardiovascular risk factors. Arch Otolaryngol Head Neck Surg 119(2): 156-161.

44. Brant LJ, Gordon-Salant S, Pearson JD, Klein LL, Morrell CH, et al. (1996) Risk factors related to age associated hearing loss in the speech frequencies. J Am Acad Audiol 7(3): 152-160.

45. Johnson AC, Bogo R, Farah A (2017) Influence of well-known risk factors for hearing loss in a longitudinal twin study. Int J Audiol 56(sup1): 6373.

46. İșlek Seçen E, Filiz Yavuz A, Levent Keskin H, Feykan Yeğin G, Müderrisoğlu $\mathrm{T}$ (2017) Effects of Maternal Smoking on Neonatal Auditory Function. Ankara Med J 17(1): 65-72.

47. Fabry AD, Davila EP, Arheart KL, Serdar B, Dietz NA, et al. (2011) Secondhand Smoke Exposure and the Risk of Hearing Loss. Tob Control 20(1): 82-85. 